\title{
ENTREVISTAS DOMICILIÁRIAS E O ENSINO E PESQUISA EM EPIDEMIOLOGIA*
}

Marilisa Berti de Azevedo Barros** José da Rocha Carvalheiro ***

BARROS, M. B. de A. \& CARVALHEIRO, J. da R. Entrevistas domiciliárias e o ensino e pesquisa em epidemiologia. Rev. Saúde públ., S. Paulo, 18:411-17, 1984.

RESUMO: São discutidas algumas características das entrevistas domiciliárias em sua utilizaçâo nas investigações sobre o processo saúde-doença e no ensino da Epidemiologia. E feito um relato sucinto de algumas experiências de pesquisa e ensino desenvolvidas no Departamento de Medicina Social da Faculdade de Medicina de Ribeirão Preto da Universidade de São Paulo-USP e no Departamento de Medicina Preventiva e Social da Faculdade de Ciências Médicas da Universidade Estadual de Campinas-UNICAMP, relacionadas a entrevistas domiciliárias. São feitas considerações sobre o elevado interesse do uso de entrevistas domiciliárias para - conhecimento da realidade de saúde dos grupos sociais que constituem a população brasileira, bem como para o desenvolvimento da própria Epidemiologia. É mostrado o enorme potencial relacionado ao ensino da Epidemiologia, a nível de graduação e pós-graduação, que pode ser desenvolvido com investigações epidemiológicas a nível da população.

UNITERMOS: Epidemiologia, ensino e pesquisa. Entrevistas domiciliárias. Morbidade.

\section{INTRODUÇÃO}

Os pesquisadores dos problemas de saúde na coletividade têm, desde longa data, utilizado informações obtidas de pacientes ou de amostras da população para conseguir realizar suas investigações e responder as suas in. quietaçōes. Não investigou Snow, naquele agosto de 1854 , em detalhe, as mortes ocorridas no início do surto de cólera do Golden Square, deixando um exemplo de investigação epidemiológica?

Embora as informações básicas dos estu. dos epidemiológicos sejam as derivadas dos registros de serviços médicos e registros de óbitos, as entrevistas realizadas com "leigos" sempre representaram um material rico para investigações na área da saúde.

$\mathrm{O}$ uso de entrevistas domiciliárias com o objetivo específico de avaliar a morbidade de uma população constitui, por sua vez, um campo de grande potencial para as investigações e para o ensino da epidemiologia. É com o intuito de estimular a discussão e o desenvolvimento desses projetos que se apresentam algumas reflexões, derivadas de experiências vividas pèlos autores, sobre investigação de condições de saúde por meio de entrevistas domiciliárias.

\footnotetext{
A MORBIDADE DA POPULAÇÃO

A utilização de entrevistas domiciliárias para investigar a morbidade da população e para o ensino de Epidemiologia, ainda que não suficientemente explorada em sua potencialidade e sistematicamente analisada, tem acumulado contribuiçðes valiosas e aberto perspectivas que a situam como uma téc-
}

\footnotetext{
*Apresentado no 1 9 Seminário Estadual de Epidemiologia e Estatísticas de Mortalidade. Teresina, Piaur, março de 1984.

**Do Departamento de Medicina Preventiva e Social da Faculdade de Ciências Médicas da Universidade Estadual de Campinas - UNICAMP - Rua Dr. Quirino, 1856 - 13.100 - Campinas, SP - Brasil.

***Do Departamento de Medicina Social da Faculdade de Medicina de Ribeirão Preto da Universidade de São Paulo - USP - "Campus" de Ribeirão Preto - 14.100 - Ribeirâo Preto, SP - Brasil.
} 
BARROS, M. B. de A. \& CARVALHEIRO, J. da R. Entrevistas domiciliárias e o ensino e pesquisa em epidemiologia. Rev. Saúde públ., S. Paulo, 18:411-17, 1984.

nica das mais promissoras para o desenvolvimento da disciplina.

Uma questão metodológica fundamental deste tipo de investigação é que desloca das instituições médicas para a comunidade, a posição de fonte central das informações so. bre saude. Este deslocamento tem várias im. plicações.

Umas delas, sempre destacada, é que a entrevista domiciliar possibilita avaliar a "morbidade geral" da população, que não poderia ser obtida da análise dos dados de óbitos ou dos registros de serviços médicos; dos óbitos, porque dependem, além da frequência das doenças, das suas taxas de letalidade, principalmente; dos registros médicos, porque sofrem as restrições, por azar existentes, na co. bertura, distribuição e qualidade dos serviços de saúde. No entanto, cabe frisar que estas fontes são preciosas para investigações clí. nicas e mesmo epidemiológicas, merecendo um esforço contínuo, conjunto, para melhoria de sua qualidade. E neste país, onde sequer as declaraçōes de óbitos atingem cobertura e qualidade suficientes, as propos. tas mais abrangentes e avançadas não devem servir de motivo para o descuido das fontes tradicionais de informações de saúde.

A entrevista domiciliar como forma de obtenção de dados de morbidade e de con. sumo de serviços de saúde, permite o acesso àquele conhecimento que fica submerso, a "morbidade percebida" ou "morbidade sentida", bem como ao conhecimento das prá. ticas não formais de restauração da saúde. Obviamente, se nem todas as pessoas pude. rem ser entrevistadas, não se conseguirá atingir o conjunto da "morbidade sentida", mas apenas aquele, cujo conhecimento é compartilhado pelo membro entrevistado da famí. lia.

De qualquer forma, a entrevista domiciliar permite esta ampliação do conhecimento do universo da morbidade no interior da população, dando a impressão de que o epidemiologista passa a ter em mãos a "verdadeira morbidade", pronta para ser mensurada.

E não é assim. À medida em que o método ultrapassa a barreira da fonte médica de dados de saúde e os obtém ao nivel da popu. lação, transforma-se o objetivo da investigação. Não são mais diagnósticos médicos a se. rem quantificados e analisados; na verdade, uma questão fundamental se impõe à refle. xão: o significado da "morbidade referida" pela população. Mais que a percepção de sinais mórbidos, as experiências de sintomas podem ser miradas, comparadas e quantificadas como se fossem "diagnósticos clí. nicos"?

Embora a percepção da doença, sua diferenciação entre grupos sociais, imprima seu efeito na demanda de serviços de saúde, esta é uma questão que não se agudiza enquanto a fonte de dados é constituída por registros médicos.

Deste modo, a morbidade percebida ou sentida apresenta-se como objeto importan. te para reflexão e investigação, especialmen. te no quadro das propostas de análise da de. terminação social do processo saúde-doença. A morbidade referida constitui dimensão operacionalizável do processo saúde-doença a ser, também, conceitualmente elaborada.

\section{A DETERMINAÇÃO SOCIAL}

Aspecto importante dos projetos de inves. tigações de saúde por meio de entrevistas do. miciliárias é que da análise de "casos clínicos", que favorece a ênfase nos aspectos biológicos, passa-se, de fato, para a análise de uma coletividade ou amostra desta, cujas ca. racterísticas são fundamentalmente sociais. Enquanto a descrição dos casos pode restringir-se a distribuições por idade, sexo, antecedentes mórbidos e outros dados, o mesmo não pode ser feito para a coletividade investigada. As informações da população sobre a presença de sintomas ou doenças e suas prá. ticas de saúde não podem ser meramente quantificadas e abstraídas da realidade em que produz e se reproduz a sociedade e da forma em que se divide o sujeito social.

Nestas investigações a inserção social das familias e grupos revela-se com muita força, não podendo ser, facilmente, marginalizada. Nas pesquisas internacionais sobre "usos de serviços de saúde", como a desenvolvida por Kohn e White ${ }^{15}$ (1976) por exemplo, as limi. tações decorrentes das comparações de da. 
BARROS, M. B. de A. \& CARVALHEIRO, J. da R. Entrevistas domiciliárias e o ensino e pesquisa em epidemiologia. Rev. Saúde públ., S. Paulo, 18:411-17, 1984.

dos médios de localidades, com estruturas sociais tão distintas, ficavam evidenciadas.

Desta forma, a passagem do estudo de "casos clínicos" para o da morbi-mortalida. de na população, tende a provocar questio. namentos sobre as análises do processo saú. de-doença feitas até então e colocam em cena reflexбes sobre a unidade de estudo: a "população". A presença das classes sociais passa então a emergir nos estudos epidemiológicos.

A utilização de entrevistas domiciliárias nas investigações de saúde coloca, também, em evidência a questão da validade e interesse das informaçб̃es do "leigo" em contrapo. siç̧̃o às informaçб̃es do médico, bem como do objeto e unidade da investigação. A possibilidade de transcender $o$ evento e $o$ indivíduo amplia a perspectiva de análise dos determinantes sociais da produção e distribuição da morbi-mortalidade.

\section{ENTREVISTADOR E ENTREVISTADO}

Outro potencial a se considerar nos inquéritos de morbidade a nível domiciliar é que embora, em muitas investigações sejam utilizados questionários fechados, num esquema analítico totalmente pré-definido pelo pesquisador, a técnica de entrevistas domiciliárias guarda o germe da abertura, no espaço da entrevista, para a visão e perspectiva do entrevistado, para o conjunto de suas representações. Podem, então, ser trazidas para o universo da pesquisa, questões não co. locadas previamente pela, equipe de pesquisadores e até mesmo por ela insuspeitadas. Só como exemplo, poder-se-ia lembrar o questionamento, aceitação, rejeição e considerações que os entrevistados emitem sobre o significado e valor da pesquisa em pauta, sobre o próprio entrevistador e inclusive sobre a figura do pesquisador.

Outra característica desse tipo de investigação é o seu dinamismo, seu caráter transformador. Pesquisadores e entrevistadores são postos em intercâmbio com as pessoas $\mathrm{e}$ fatos investigados, num espaço econômico e político que não pode ser ignorado. $E$, ainda que em menor intensidade, também os entre. vistados são solicitados a refletir, avaliar, a "ter voz". Pesquisa e pesquisadores se trans. formam no decorrer dela. Essa vitalidade, es. sa permeabilidade, essa transformaçăo inerente à técnica, provocada por uma relação mais próxima entre objeto investigado e pesquisadores, confere a essa forma de investigação uma feição didática e põe à tona sua dimensão política.

Esse potencial das investigações de saúde por meio de entrevistas domiciliárias, num momento em que crescem as inquietudes para desvendar e aprofundar o conhecimento sobre as relaçōes do social com o processo saúde-doença e práticas de saúde, precisa ser explorado mais insistentemente e utilizado, também, nas práticas de ensino da Epidemiologia.

\section{INQUERITO DE MORBIDADE E ENSI- NO DE EPIDEMIOLOGIA: A EXPERIEN. CIA DE RIBEIRÃO PRETO.}

$O$ relato, ainda que breve, de algumas experiências vividas em Ribeirão Preto e outras ainda incipientes, em Campinas, são úteis para situar alguns pontos sobre a operacionalização desses projetos bem como sobre os desdobramentos que podem ter a nível do ensino da Epidemiologia.

Em Ribeirão Preto, o projeto desenvolvido a partir de 1974 , foi precedido por duas investigações de menor porte realizadas, uma na Vila Guatapará - sede de um distrito do município de Ribeirão Preto - em 1972, e outra na Vila Lobato, bairro da cidade de Ribeirão Preto, em 1973.

A metodologia empregada nesses dois projetos e os resultados gerais obtidos, já se encontram publicados ${ }^{9}, 10,11,12$.

Vale destacar que enquanto desenvolvimento de um projeto de investigação epidemiológica (levantamento de condições de saúde e pesquisa epidemiológica específica), essas experiências foram fundamentais para apurar os instrumentos, métodos e técnicas específicas de coleta e análise dos dados. Foram importantes na germinação e maturação do Projeto de Investigação de Morbidade e Uso de Serviços de Saúde por meio de Entre. vistas Domiciliárias de Ribeirão Preto, cami- 
BARROS, M. B. de A. \& CARVALHEIRO, J. da R. Entrevistas domiciliárias e o ensino e pesquisa em epidemiologia. Rev. Saúde públ., S. Paulo, 18:411-17, 1984.

nhando no sentido de uma complexidade crescente na amostragem, na estruturação do questionário e no treinamento e padroniza. ção dos entrevistadores.

Enquanto na Vila Guatapará todas as 330 casas foram investigadas e, na Vila Lobato, $50 \%$ das 924 casas, resultado de uma amostragem casual simples, a obtenção de uma amostra semanal de $1 \%$ dos domicilios de Ribeirão Preto exigiu técnicas complexas de amostragem $^{8}$.

Quanto ao treinamento dos entrevistadores e as características destes, no projeto de Guatapará, ainda não havia um manual de preenchimento do questionário, enquanto que o manual do projeto de Ribeirão Preto atingiu grande detalhamento. De algumas poucas reuniões para o treinamento (no projeto Guatapará) chegou-se, no de Ribeirão Preto, a uma prolongada e cuidadosa preparação dos entrevistadores ${ }^{5}$.

Com 100 estudantes de 30 ano de Medicina atuando como entrevistadores na primeira experiência, e poucos alunos de enfermagem e alunos de pós-graduação na segunda (10 entrevistadores), no projeto de Ribeirão Preto já se dispõe de uma equipe de 13 entrevistadoras contratadas e treinadas especifica. mente para esta atividade.

O próprio questionário sofre mudança radical nessa 3 ạ fase em relação às anteriores, que vem propiciar ao sistema sua grande versatilidade. Versatilidade que pode ser avaliada pela variedade de temas incluídos em uma "Apresentação Coordenada", realizada na 30a Reunião Anual da SBPC, em $1978^{1,6,7,17,22}$.

Por outro lado, em relação ao ensino da epidemiologia, os diferentes projetos tiveram características diversas. $O$ de Guatapará ser. viu de prática de levantamento de dados a alunos de 30 ano de medicina, no interior de um curso de Parasitologia. A realização da entrevista domiciliar, da verificação do nível de vida, das condições da habitação e dos quintais, da criação de animais e procedência dos alimentos, permitia entender e discutir os resultados dos exames coprológicos numa análise mais abrangente e integrada na realidade sócio-econômica daquela população.
No projeto de Vila Lobato, a experiência de entrevistador é vivenciada por alunos de enfermagem em estágio de saúde pública $\mathrm{e}$ por alunos de pós-graduação, servindo agora, de preparo e treinamento para pessoal que vai se voltar a práticas de saúde na coletivi. dade e a práticas de investigações.

No 30 momento, quando a execução das entrevistas e a manutenção do sistema de coletas de dados independe de uma integração com o ensino da epidemiologia, verificase que ela se mantém principalmente a nível do curso de pós-graduação.

A disciplina de "Amostragem" da área de bioestatística passa, a partir de 1978, a ser oferecida também aos pós-graduandos da área de medicina preventiva e é criada a disciplina "Métodos de Inquérito em Investigação Epidemiológica". Essas disciplinas permitiram a integração, em projetos ligados ao Sistema de Amostragem e Coleta de Dados, dè pós-graduandos com formações diversas e com evidentes vantagens para o conjunto dos alunos.

Com base no Sistema de Investigação de Morbidade e de sua infra-estrutura, pós-gra. duandos de medicina preventiva elaboraram projetos de teses de mestrado e doutoramento $3,4,13,16,18,23$.

A nível de graduação, o projeto representou uma fonte de dados atualizados para trabalhos, exercícios e análises. Constituiu, também, material de análise para projetos de iniciação científica de alunos interessados na área de medicina preventiva e social ${ }^{1}, 2,14$, 19,21 .

\section{ALGUMAS EXPERIENCIAS EM CAMPI- NAS}

De menor porte e ainda incipientes, algumas experiências vividas em Campinas serão brevemente relatadas, indicando as alternativas que têm sido buscadas.

No segundo semestre de 1979 , os alunos do Curso de Enfermagem da Faculdade de Ciências Médicas da Universidade Estadual de Campinas-UNICAMP desenvolveram um levantamento sobre condições de saúde e prevalência de parasitoses em uma amostra 
BARROS, M. B. de A. \& CARVALHEIRO, J. da R. Entrevistas domiciliárias e o ensino e pesquisa em epidemiologia. Rev. Saúde públ., S. Paulo, 18:411-17, 1984.

da população de Monte Mór*. Trata-se de município pequeno (com 9.826 habitantes em 1979), próximo a Campinas $(20 \mathrm{~km})$, cujo prefeito havia solicitado uma avaliação ao Departamento de Medicina Preventiva e Social da UNICAMP. A Prefeitura contribuiu com veículo para transportar os alunos aos bairros mais distantes. Um ônibus pertencente ao Centro de Saúde Escola de Paulínia transportou alunos e supervisores a Mon. te Mór.

O questionário, o manual de preenchimento e algumas observações sobre técnicas de entrevistas foram discutidos com os alunos antes do trabalho de campo. O levanta. mento dos dados foi realizado em 4 perío. dos, um por semana. Embora os alunos não tenham tido tempo suficiente para analisar os dados, e se revelassem questionáveis os resultados obtidos dos exames coprológicos, o trabalho constituiu experiência didática bas. tante interessante, tendo o curso sido ava. liado positivamente pelos alunos.

No segundo semestre de 1980, também no Curso de Epidemiologia para enfermagem, os alunos, em grupos, realizaram visitas a grupos de familias, nas quais haviam ocorrido casos de doenças de notificação compulsória. Sem pretender um levantamen. to completo dos casos, a experiencia foi didaticamente feliz. As visitas favoreceram e estimularam o estudo e discussão dos aspectos epidemiológicos das doenças seleciona. das.

Em 1983, inicia-se no Departamento de Medicina Preventiva e Social da Faculdade de Ciências Médicas da Universidade Esta. dual de Campinas-UNICAMP, o trabalho de campo do Projeto "Mortalidade e Classe Social: um estudo no município de Campinàs", financiado pela FINEP. Com o objetivo de caracterizar o perfil da mortalidade nas frações de classes sociais, o projeto implica a organização de uma infra-estrutura que permita a coleta das declarações de óbitos, o sorteio de uma amostra semanal e a realiza. ção das entrevistas domiciliárias junto aos familiares dos óbitos selecionados ${ }^{20}$.
Aproveitando a estrutura montada para este Projeto, um grupo de 14 alunos do 2 ? ano de medicina da UNICAMP, realiza, no segundo semestre de 1983, um estudo sobre os óbitos infantis ocorridos em Campinas, em agosto daquele ano* O estudo constitui um dos projetos desenvolvidos como "parte prática" da Disciplina "Ciências Sociais aplicadas à Medicina". O questionário bem co. mo todas as etapas da pesquisa foram discu. tidos e decididos com o grupo de alunos. As familias onde ocorreram os óbitos deveriam ser entrevistadas e, ao lado de uma análise quantitativa dos dados, os alunos fizeram uma análise "qualitativa", discutindo a situa-. ção de vida daquelas familias, suas histórias de migração e ocupação, relatando com mais detalhe os casos que os "marcaram" e analisando, inclusive, o valor deste tipo de pes. quisa, na vivência do estudante de medicina.

Constituíram, sem dúvida, experiências estimulantes que motivam a continuidade da busca de formas de ensinar epidemiologia associando a discussão dos conceitos e técnicas às práticas de investigação.

\section{CONSIDERAÇÕES SOBRE O USO DE ENTREVISTAS DOMICILIÅRIAS NO EN. SINO DE EPIDEMIOLOGIA}

Desse conjunto de experiências vividas, não suficientemente analisadas e sistematizadas, busca-se avançar algumas observações.

O ensino da epidemiologia torna-se mais motivador e mais abrangente à medida em que os alunos têm a possibilidade de viven. ciar alguma forma de pesquisa. Por outro la. do, e especialmente tratando-se de alunos de graduação, essa prática é muito mais gratificante quando possibilita o "contato com a realidade" (na expressão dos alunos), do que quando utiliza dados secundários.

Mas, a inserção dos alunos em pesquisa é questão complexa e controversa. Questiona-se o significado dessa experiência: tem valor apenas como técnica didática ou é possível realizar, com alunos, uma pesquisa com "va. lidade científica"?

Também se indaga sobre a validade de

* Dados inéditos 
BARROS, M. B. de A. \& CARVALHEIRO, J. da R. Entrevistas domiciliárias e o ensino e pesquisa em epidemiologia. Rev. Saúde públ., S. Paulo, 18:411-17, 1984.

inserir o aluno apenas na fase de coleta de dados, de pesquisa já planejada e definida em seus objetivos. Os alunos deveriam participar em todas as fases do projeto, quando possível. Mas, nessa situação seria exequivel a realização completa da pesquisa? Seria possível conseguir o treinamento e padronização ne. cessários?

Evidentemente o caráter de cada curso e suas limitaçðes, sejam de carga horária, perríodos e horários, número de alunos, local, recursos disponíveis e outras, vão definir as possibilidades dessa inserção dos alunos em investigaçōes e o caráter dessas investigações. De qualquer forma, uma experiência de in. vestigação na comunidade é melhor que ne. nhuma e é sempre possível buscar a pesquisa possível nos limites de cada curso.

A localização das Universidades e Faculdades de Medicina em grandes centros urbanos coloca acessível aos estudos uma realidade sanitária que sofre o impacto da migração e urbanização intensas, das profundas desigualdades sociais e da trágica situação dos serviços de saúde. Desta forma parece mais adequada a investigação que, ao lado do "quantitativo", abra espaço para o "qualitativo".

As entrevistas domiciliárias como técnica de investigação-nsino permitem detectar e refletir sobre a relação da organização social com a questão da saúde. Possibilitam análises sobre a distribuição da morbi-mortalida. de por bairros e grupos sociais. Permitem a reflexão sobre a percepção e concepções populares da doença e o conhecimento das consideraçōes e críticas da população com res. peito aos serviços de saúde.

Representam, nesta perspectiva, um instrumento do maior valor para a ampliação do conhecimento do processo saúde-doença na coletividade e para a formação de profissionais da área da saúde.

BARROS, M. B. de A. \& CARVALHEIRO, J. da R. [Household surveys as related to epidemiological teaching and research]. Rev. Saúde públ., S. Paulo, 18:411-17, 1984.

ABSTRACT: Some aspects of the use of household surveys in research into the health-sickness process and in the teaching of ' Epidemiology are discussed. A brief report is made of some experiments : in teaching and research, related to household surveys, carried out by the Department of Social Medicine of the School of Medicine of Ribeirão Preto, University of S. Paulo and by the Department of Preventive and Social Medicine of the School of Medical Sciences, University of Campinas. The great potencial of this type of investigation for the knowledge of the health conditions of social classes and for the development of Epidemiology is pointed out by the authors. The importance of these surveys for the teaching of Epidemiology for graduate and post-graduate students is emphasized. dity.

UNITERMS: Epidemiology, research and teaching. Household interview methods. Morbi-

\section{REFERENCLAS BIBLIOGRÅFICAS}

1. ALVES, M. L. D. \& CARVALHEIRO, J. da R Epidemiologia dos fenômenos para-normais durante um programa de televisão ("Uri Geller"). Cienc. Cult., 30 (Supl.): 76-7, 1978.

2. ALVES, M. L. D. \& CARVALHEIRO, J. da R. Classificação das morbidades referidas em entrevistas domiciliárias. Cienc. Cult., 31 (Supl.): 83, 1979.

3. BARRETO FILHO, A. D. Aspectos epidemiológicos da sífilis: Vila Virginia, Ribeirão Preto. Ribeirão Preto, 1980. [Dissertação de Mestrado - Faculdade de Medicina de Ribeirāo Preto da USP]

4. BARROS, M. B. de A. Saúde e classe social: um estudo sobre morbidade e consumo de medicamentos. Ribeiräo Preto, 1983. [Tese de Doutoramento - Faculdade de Medicina de Ribeirão Preto da USP]

5. CARVAlHeiro, J. da R. Levantamento de condições de saúde por entrevistas domiciliárias. Ribeirão Preto, 1975. [Tese de Livre Docência - Faculdade de Medicina de Ribeirão Preto da USP] 
BARROS, M. B. de A. \& CARVALHEIRO, J. da R. Entrevistas domiciliárias e o ensino e pesquisa em epidemiologia. Rev. Saúde públ., S. Paulo, 18:411-17, 1984.

6. CARVALHEIRO, J. da R. \& CARVALHEIRO, C. D. G. Medidas de morbidade produzidas por duas fontes diversas: entrevistas domiciliárias e egressos hospitalares. Cienc. Cult., 30 (Supl.): 74, 1978.

7. CARVALHEIRO, J. da R. \& PINA, R. M. S. Montagem e utilização de um sistema contínuo de entrevistas domiciliárias para investigação epidemiológica. Cienc. Cult., 30 (Supl.): 77, 1978.

8. CARVALHEIRO, J, da R. \& SANCHES, $O$. Amostragem domiciliar contínua em estudos epidemiológicos e no ensino. Rev. Saúde públ., S. Paulo, 13: 195-202, 1979.

9. CARVALHEIRO, J. da R. et al. Levantamento de condições de saúde por entrevistas domiciliárias. III - Vila Guatapará: Metodologia, características da família e seu domiculio. Medicina, Ribeirāo Preto, 15:13 $-25,1982$.

10. CARVALHEIRO, J. da R. et al. Levantamento de condições de saúde por entrevistas domiciliárias. IV - Vila Guatapará: Características individuais e morbidade referida. Medicina, Ribeirão Preto, 15:27-37, 1982.

11. CARVALHEIRO, J. da R. et al. Levantamento de condiçōes de saúde por entrevistas domiciliárias. V - Vila Lobato: Metodologia, características da famflia e seu domicílio. Medicina, Ribeirão Preto, 15:57-71, 1982.

12. CARVALHEIRO, J. da R. et al. Levantamento de condições de saúde por entrevistas domiciliárias. VI - Vila Lobato: Características individuais e morbidade referida. $\mathbf{M e}$. dicina, Ribeirão Preto, 15:139-53, 1982.

13. FORSTER, A. C. Cobertura dos serviços de imunização em Ribeirâo Preto, através de entrevistas domiciliárias. Ribeirão Preto, 1979. [Dissertação de Mestrado - Faculdade de Medicina de Ribeirāo Preto da USP]

14. GRIGOLETTO, N. M. \& PEREIRA, J. C. Coeficientes de morbidades referidas em $\mathrm{Ri}$ beirão Preto (SP). Cienc. Cult., 31(Supl.): 85-6, 1979.

15. KOHN, R. \& WHITE, K. L., ed. Health care: on international study. London, Oxford University Press, 1976.
16. MARZOCHI, M. C. A. Estudo epidemiológico da poluição por enteroparasitas em áreas de horticultura da cidade de Ribeirão Preto, SP, Brasil. Londrina, 1975. [Tese de Doutoramento - Centro de Ciências da Saúde - Universidade de Londrina]*

17. MATTAR, $\mathbf{N}$. Incidência de meningites em diversos grupos da população de Ribeirão Preto. Ribeirão Preto, 1978. [Dissertação de Mestrado - Faculdade de Medicina de Ribeirão Preto da USP].

18. MATTAR, N. \& CARVALHEIRO, J. R. Incidência de meningite em diversos grupos da população de Ribeirão Preto. Ciêenc. Cult., 30 (Supl.): 74, 1978.

19. MUSSI, M. M. \& PEFEIRA, J. C. Coeficientes de consultas médicas referidas em Ribeirão Preto (S. P.). Ciênc. Cult., 31 (Supl.): 85, 1979.

20. NUNES, E. D. \& BARROS, M. B. A. Mortalidade e classe social: um estudo no municipio de Campinas. Campinas, UNICAMP, 1981. [mimeografado].

21. OLIVEIRA, G. G. \& CARVALHEIRO, J. R. Distribuição dos graus de incapacitação em pessoas que referem episódios de morbidade. Ciênc. Cult., 31 (Supl.): 83, 1979.

22. PEREIRA, J. C. \& CARVALHEIRO, J. R. Morbidade, conduta adotada e consultas médicas, por estratos sociais em Ribeirão Preto (S. P.). Ciênc. Cult., 30 (Supl.): 77, 1978.

23. RAMOS, M. C. Levantamento da prevalência de doenças respitatórias obstrutivas crônicas na cidade de Ribeirão Preto - São Pau10. Kibeirão Preto, 1980. [Tese de Doutoramento-Faculdade de Medicina de Ribeirãon Preto da USP].

* Nota: descreve levantamentos anteriores à experiência de Guatapará, 1972.

Recebido para publicaç̄o em 28/06/1984 Aprovado para publicafäo em 17/08/1984 